\title{
Observations on the ontogeny of saccharine preference in the neonate rat
}

\author{
Harry L. Jacobs \\ UNIVERSITY OF ILLINOIS
}

\begin{abstract}
Acceptability of saccharine, lactose, and water was measured in rats 0-21 days of age. Although saccharine acceptability increased over the testing period, lactose and water did not. The results are not in agreement with a reinforcement interpretation of saccharine preference.
\end{abstract}

\section{Problem}

Adult rats show a preference for non-nutritive saccarine solutions (Hausmann, 1933; Beebe-Center, et al., 1948). Although this phenomenon has been variously attributed to drive reduction (Miller, 1951), secondary reinforcement (Osgood, 1953), consummatory responses (Sheffield, et al., 1950; 1954), and innate palatability for "sweets" (Woodworth \& Schlosberg, 1954; Young, 1961), study of the adult animal has not allowed an unequivocal choice among these alternatives.

Nachman (1957), has attempted to separate innate from environmental determinants of saccharine preference by limiting the opportunity to taste sweet substances in young animals. Newly weaned rats (25 days of age) raised on a carbohydrate-free diet showed adult saccharine preference equivalent to animals raised on a very sweet diet. This result suggested that previous experience with a sweet substance was unnecessary for the development of saccharine preference in the adult rat. However, this conclusion is open to question, since the critical learning may have already taken place during the 25-day weaning period before Nachman's experiment started.

The conditions of nurturance in the infant rat are ideally suited for the application of the principle of secondary reinforcement to the origin of saccharine preference. Rat milk contains $2.8 \%$ lactose (milk sugar). Thus, repeated association of this initially neutral sweet taste with hunger reduction should accumulate secondary incentive value for sweet substances, generalizing to saccharine in the adult rat. This argument was evaluated by direct observation of sweet preferences in neonate rats. The present experiment is the first of a series on the ontogeny of sugar preference, comparing the development of acceptability of a sweet saccharine solution, normally preferred by adult rats, to a less sweet lactose solution, equivalent in concentration to that contained in rat's milk.

\section{Method}

Four litters of Holtzman rats were used as Ss. Within $4 \mathrm{hr}$. after birth the litters were reduced to the six heaviest animals, identified by application of dilute gentian violet. Mothers were maintained on Wayne Lab Blox and water; sawdust litter was used. Each neonate was given four daily liquid acceptability tests, at 9 a. m., 1 p. m., 5 p. m., and 9 p. m. until they were 21 days old. Two Es were used, alternating test runs.

At each test period the litter was removed and put into a small cardboard box, beneath a 60-watt lamp for warmth. All Ss in a litter were tested consecutively on one liquid, returned to the box if a second run was planned, and to the mother when the last run was complete. On each test, $\mathrm{E}$ held $\mathrm{S}$ in his left hand with its jaws resting between thumb and forefinger. Liquids were applied with a 2 cc syringe tipped with a 1-in length of PE-10 tubing. The tube was gently inserted into Ss mouth and a single drop $(.01 \mathrm{ml})$ placed upon the tongue. The tube was then withdrawn and held about $1 / 4$ in away while the response was observed. Preliminary tests showed a tendency to lick initially at the tube regardless of contents. Thus, the tube was reinserted 4-5 times with every solution before a rating was applied to the trial. A single trial (one S, one liquid) took about $35 \mathrm{sec}$.

A four category rating scale was used to evaluate acceptability on each trial: (1) maximum negative; $S$ struggles, vocalizes, turns and twists head, rapid extrusion of liquid and tube. (2) medium negative; $\mathrm{S}$ is passive, no swallowing, mouth open, tongue retrudes, liquid dribbles out of mouth. (3) medium positive; S swallows solution, very little overt licking. (4) maximum positive; S sucks overtly, swallows solution, reaches out for tube when slowly removed, continues licking lips after swallowing.

Test liquids were allowed to come to room temperature $\left(78^{\circ} \mathrm{F}\right)$ before each test series. The liquids were kept in bottles identified by arabic letters. E applied the test liquids for each run in a predetermined random order, without knowledge of the contents of each bottle. distilled water was used in mixing solutions and as a test liquid.

\section{Results and Diseussion}

Figure 1 shows the results of two replications of this experiment, the upper portion (A) on three litters, each tested on a single solution, and the lower portion (B) on a single litter tested on three solutions. The results were similar in both cases, saccharine increasing over the testing period, and clearly more acceptable than lactose and water, which were indistinguishable from one another, both rising only slightly beyond 2.0 , the neutral point on the acceptability scale. Three more litters have been tested on saccharine since these data were collected. The overall results suggests that the sharp rise on days $7-9$ on the lower portion of the 


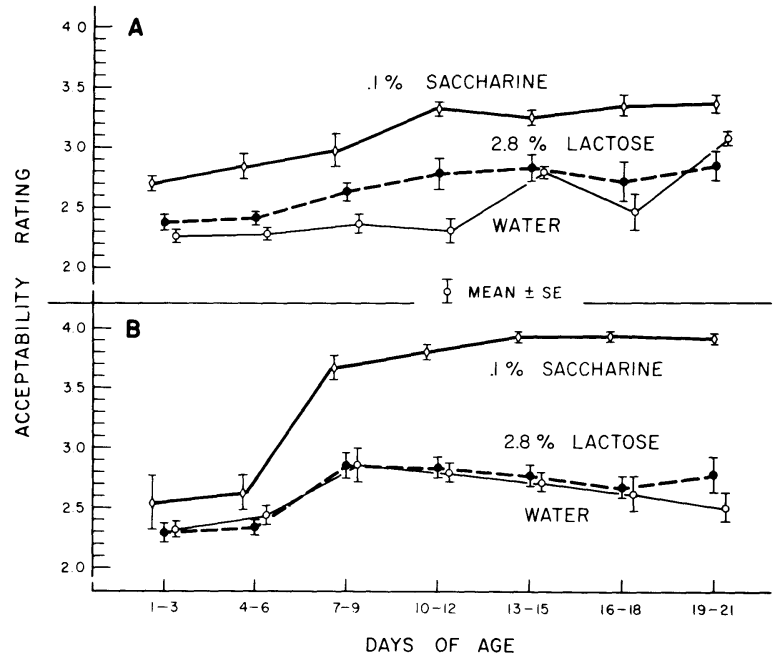

Fig. 1. Preweaning acceptability of lactose and saccharine solutions, and water. A. Independent litters containing 5 pups each. B. Single 4 pup litter, tested on all solutions.

figure (B) is more typical than the gradual increase shown in the upper portion (A).

The increase in saccharine acceptability might be taken as a conditioning curve reflecting an increase in its incentive value, generalizing from the sweetness of the mother's milk, the original point of reinforcement. However, if sweetness preference is acquired via secondary reinforcement, one would expect both sweet solutions to increase with postnatal age, the sweeter saccharine solution lagging behind the lactose solution, which is closer to the point of primary reinforcement (milk) on the generalization gradient. These data are in direct opposition to this prediction. Thus, it is concluded that the ontogeny of saccharine preference cannot be explained by secondary reinforcement in simple associative learning.

Further analysis of the methods as well as the basic phenomenon show that it is possible to use as many as four trained Es on each litter without significantly affecting reliability, and that presentation of empty tubes to the pup does not elicit significant acceptability. Current experiments are evaluating sweetness and state of hunger as parameters in the development of saccharine preference.

\section{References}

BEEBE-CENTER, J. G., BLACK, P., HOFFMAN, A. C., \& WADE, M. Relative per diem consumption as a measure of preference in the rat. J.comp.physiol. Psychol., 1948, 41, 239-251.

HAUSMANN, M. F. The behavior of albino rats in choosing foods: Differentiation between sugar and saccharine. J. comp. physiol. Psychol., 1933, 15, 419-428.

MILLER, N. E. Comments on multiple-process conceptions of learning. Psychol. Rev., 1951, 58, 375-381.

NACHMAN, M. The influence of diet and age on saccharine preference in rats. Amer. Psychol., 1957, 12, 461. (Abstract)

OSGOOD, C. Method and theory in experimental psychology. New York: Oxford, 1953. Pp. 439-440.

SHEFFIELD, F. D., \& ROBY, T. B. Reward value of a non-nutritive sweet taste. J.comp.physiol. Psychol., 1950, 43, 471-481.

SHEFFIELD, F. D., ROBY, T. B., \& CAMPBELL, B. A. Drive reduction versus consummatory behavior as determinants of reinforcement. J. comp. physiol. Psychol., 1954, 47, 349-354.

WOODWORTH, R. S., \& SCHLOSBERG, H. Experimental psychology (rev. ed.). New York: Holt, 1954. P. 684.

YOUNG, P. T. Motivation and emotion. New York: Wiley, 1961. Pp. 248-251.

\section{Note}

1. This work was supported by a Public Health Service Grant MH05754 and grant-in-aid from the Corn Products Institute of Nutrition. I am pleased to acknowledge the help of L. J. Goldsmith and R. P. Kesner, who worked as experimenters in the acceptability tests. 